\title{
EVIDENCE THAT WOLF-RAYET STARS ARE PRE-MAINSEQUENCE OBJECTS
}

\author{
ANNE B. UNDERHILL \\ Department of Geophysics and Astronomy \\ University of British Columbia \\ Vancouver, B. C., V6T 1W5, Canada
}

\begin{abstract}
The evidence is reviewed that Population I Wolf-Rayet stars have solar abundances, that they are surrounded by remnant disks formed from their natal clouds, and that their rate of mass loss is moderate. These properties are consistent with Wolf-Rayet stars being young objects recently arrived on the main-sequence rather than the evolved, peeled-down remnants of massive stars.
\end{abstract}

\section{Introduction}

Many people believe that Population I Wolf-Rayet stars are highly evolved massive stars which show anomalous abundances on their surfaces as a result of mass loss at a rate of the order of $5 \times 10^{-5} M_{\odot} \mathrm{yr}^{-1}$. I shall present evidence (1) that they have solar abundances, (2) that they are surrounded by remnant disks formed from their natal cloud, and (3) that their rate of mass loss is uncertain, but possibly of the order of or smaller than $10^{-6} M_{\odot} \mathrm{yr}^{-1}$. These properties together with the fact that Wolf-Rayet stars are associated with $O$ and early $B$ stars in regions which radiate interstellar $C O$ lines points toward them being young objects with ages no greater than about $5 \times 10^{6}$ yrs.

The predominant emission-line spectra of Wolf-Rayet stars are suggestive of the spectra of Herbig Ae/Be stars and T Tauri stars. The major difference is that in the line-emitting regions (LERs) of Wolf-Rayet stars the electron temperature $\left(T_{e}\right)$ is of the order of $10^{5} \mathrm{~K}$ whereas in the case of the less massive pre-mainsequence stars it is of the order of or less than $10^{4} \mathrm{~K}$. To generate a high $T_{e}$ in the LER of a Wolf-Rayet star one may postulate that mechanical energy is transformed to heat by MHD effects which may occur in a lowbeta plasma. Whenever larger than normal magnetic fields are occluded as a rnassive star $\left(M_{*} \geq 10 M_{\odot}\right)$ is formed, a high excitation, emission line spectrum may be generated as a result of the deposit of non-radiative energy and momentum in the LER. Such spectra are the criteria by which Wolf-Rayet stars are recognized. Analysis of these spectra reveals the physical state of the LER, not of the underlying photosphere. Additional information and postulates must be made to relate the underlying stars to the theory of evolution of massive stars. These postulates and information are different for Population I than for Population II Wolf-Rayet stars.

Study of binary Wolf-Rayet stars and of those in clusters shows that typically Population I Wolf-Rayet stars have masses of the order of $10-15 M_{\odot}$ and that their luminosities are typical of hydrogen-burning stars of these masses. Their effective temperatures estimated from integrated fluxes (Underhill 1983) and from the radiation temperatures $\left(T_{*}\right)$ which generate their spectra (Bhatia and Underhill 1986, 1988) are appropriate for their 
masses, i.e. of the order of $25,000-30,000 \mathrm{~K}$.

The Population I Wolf-Rayet stars have 6-cm fluxes of the order of or less than a mJy, and all show significant infrared excess fluxes. These properties suggest that the Population I Wolf-Rayet stars are buried in much circumstellar plasma. Many show significant polarisation, see, for example, St. Louis et al. (1987) and Drissen et al. (1987). This indicates that a Wolf-Rayet star is buried in a cloud of electrons which is not spherically symmetric. Binary Wolf-Rayet stars tend to show polarisation changes which correlate with the orbital period, see, for instance, Drissen et al. (1986), St. Louis et al. (1988), as well as Schulte-Ladbeck and van der Hucht (1989). Some Wolf-Rayet stars show small random changes in polarisation.

\section{The Analysis of the Emission Lines of Wolf-Rayet Spectra}

The first challenge is to find what range of parameters is significant for creating WolfRayet type spectra. I use the one-representative-point theory of Castor and van Blerkom (1970) for a wide range of parameters to predict the relative energies radiated in lines in the visible spectral range for comparison with observations. This theory sets up the equations of statistical equilibrium for a model atom and provides expressions for the needed radiation field in the case where a velocity gradient exists in the LER. All emission lines are assumed to be formed by photons which escape from the same body of plasma.

It is essential to carry terms in the equations of statistical equilibrium for all types of radiative and collision transitions which occur in a model atom as a result of the presence of the atom in the ambient radiation field of an LER. Bhatia and Underhill $(1986,1988)$ tell how to evaluate photoionization from and radiative recombination rates to all levels of the model atoms, rates for collisional ionization and three-body recombination, also photo and collision excitation rates via line transitions and their reverse rates, and how to estimate the effect of dielectronic recombination on the degree of ionization of each atom/ion sensitive to dielectronic recombination.

The "Of" emission lines are strong in Wolf-Rayet spectra. It is now known that these lines are sensitive to the selected cascade routes which may follow dielectronic recombination. It has been impossible so far to carry such details in the equations of statistical equilibrium for simple model Wolf-Rayet LERs. Because the relative intensities of "Of" emission lines to other lines have been empirically selected as criteria for the various WC and WN subtypes, it is impossible at this time to predict patterns of line emission to match what is observed for the various subtypes. However, it has been possible for Bhatia and Underhill to establish the major properties of WC and WN LERs from their studies of the statistical equilibrium of $\mathrm{H}, \mathrm{He}, \mathrm{C}, \mathrm{N}$, and $\mathrm{O}$ model atoms under a wide range of conditions and for several compositions, see BU86, BU88, and BU89.

The results are as follows:

QUANTITY

Radiation temperature, $T_{*}$

Electron temperature, $T_{e}$ :

Electron density, $N_{e}$ :

Composition:
WC LER

$25,000 \mathrm{~K}$

$5 \times 10^{4} \leq T_{\mathrm{e}} \leq 10^{5} \mathrm{~K}$

$10^{10}-10^{11} \mathrm{~cm}^{-3}$

solar

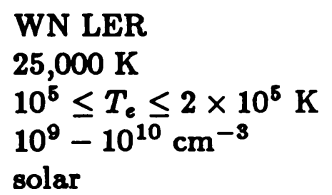

WN LER

$10^{5} \leq T_{e} \leq 2 \times 10^{5} \mathrm{~K}$

solar 
These parameters allow one to predict successfully the observed relative intensities in many of the key lines of Wolf-Rayet spectra. See the papers by Bhatia and Underhill for details. The energy of the electrons in the LER, thus the electron temperature, is the dominant factor which causes WC spectra to be different from WN spectra. Density is a second important parameter.

\section{Discussion}

Five properties of atmosphere models appropriate for Wolf-Rayet stars can be inferred by studying the results of the one-point analyses carried out by Bhatia and Underhill.

\subsection{SOLAR ABUNDANCES}

Previous conclusions that anomalous abundances are present on the surfaces of Wolf-Rayet stars are a result of the use of incomplete equations of statistical equilibrium or of the assumption of LTE and of failure to explore a wide enough range of $T_{*}, T_{e}$, and $N_{e}$ such that the true pattern of predicted line intensities as functions of the parameters can be seen. Bhatia and Underhill $(1986,1988,1989)$ have discussed the strengths and limitations of their studies. All in all, good agreement with key line ratios used to classify Wolf-Rayet spectra is obtained. The few discrepancies can be understood in terms of the neglect by Bhatia and Underhill of the details of dielectronic recombination, and the inadequate treatment of detailed balance in a few resonance lines. If it was all right to neglect photoionization from and radiative recombination to excited levels, the results of BU88 and BU89 would have confirmed those obtained in the NLTE studies by others. These results were not confirmed. Local thermodynamic equilibrium is not a viable hypothesis for line formation in Wolf-Rayet LERs.

\subsection{SIZE AND SHAPE OF THE LER}

BU88 find that the emissivity in He II $\lambda 5411$ of typical Wolf-Rayet LERs is such that the LER has a volume of $10^{43}-10^{44} \mathrm{~cm}^{3}$. Because a contiguous sphere of this volume at the typical $N_{e}$ in an LER is nearly opaque in electron scattering, (which would imply larger apparent optical continuum radii for Wolf-Rayet stars than are estimated, see Underhill 1983), BU88 suggest the LER may be a thin ring-like disk at a radius of about $10^{15} \mathrm{~cm}$. Evidence that line formation in a rotating, thin ring occurs is given by the observed profile of He I $\lambda 5876$ in HD 191765, WN6. This line has an unchanging double-peaked shape typical of line formation in a ring-like disk where the lines are broadened by macroturbulence, see Underhill et al. (1990).

\section{3 $T_{e}$ IN AN LER}

A high value of the order of $10^{5} \mathrm{~K}$ requires the deposit of non-radiative energy in the LER. BU88 suggest that turbulent mechanical energy in the disk is transformed to heat by MHD effects in the presence of a small magnetic field of interstellar origin. A field of 4-8 gauss is sufficient to create a low-beta plasma which could be heated by MHD effects.

\subsection{LOW RATE OF MASS LOSS}

If the observed 6-cm fluxes of Wolf-Rayet stars are transformed to rates of mass loss assuming thermal bremsstrahlung in a spherical wind, there is a shortfall of radiative 
momentum to drive the wind by a factor of 20-50. Underhill (1984) has suggested that part of the 6-cm flux could be due to gyroresonance magnetic bremsstrahlung. Some of the 6-cm flux could also be generated in a disk-driven wind, cf. Pudritz and Norman (1986). Consequently the true $\dot{M}$ from the star is probably much less than $5 \times 10^{-5} M_{\odot} \mathrm{yr}^{-1}$. The fact that sharp subpeaks are seen on the profile of He II $\lambda 5411$ in the spectrum of HD 191765 (Moffat et al. 1988; Underhill et al. 1990) indicates that this star is not surrounded by a dense sphere of electrons. If it were, the subpeaks would be greatly broadened by electron scattering. The rate of mass loss from a Wolf-Rayet star alone may be $\leq 10^{-6} M_{\odot}$ $\mathrm{yr}^{-1}$.

\section{$3.5 T_{\text {eff }}$ OF WOLF-RAYET STARS}

The calculations of BU86, BU88, and BU89 clearly indicate that the radiation temperature $\left(T_{*}\right)$ of the continuous spectrum shortward of the Lyman limit is about 25,000 K. Model atmospheres for massive stars of solar composition indicate that this condition is met for $T_{\text {eff }}=25,000-30,000 \mathrm{~K}$. Such effective temperatures are appropriate for stars having the masses of Population I Wolf-Rayet stars. Solutions of the radial-velocity orbit of the eclipsing Wolf-Rayet binary CQ Cephei (Underhill, Gilroy, and Hill 1990) show that an acceptable solution can be obtained for stars having $M_{W R}=13.6 M_{\odot}$ and $M_{\text {comp }}=16.0 M_{\odot}$. Both stars are in the hydrogen-burning stage. If the Wolf-Rayet star was an evolved object such as postulated by Maeder and Meynet $(1987,1988)$ and by Langer (1989), its surface abundances would be anomalous and its effective temperature would be about $1.4 \times 10^{5} \mathrm{~K}$. The effective temperature of the companion star would be about $30,000 \mathrm{~K}$. Such effective temperatures would not produce nearly equally deep primary and secondary light minima as are observed from far ultraviolet to visible wavelengths (Stickland et al. 1984). The high effective temperatures of the evolved model stars of Maeder and Meynet (1988) and of Langer (1989) make these models unsuitable for representing the Wolf-Rayet star of CQ Cephei and, for that matter, of V444 Cygni. The known properties of the light curves cannot be reproduced at $1300 \AA$ and at $5500 \AA$ when one model star is taken from the sets of highly evolved, peeled down models of Maeder and Meynet or of Langer, and the other from sets of hydrogen-burning models.

\section{Conclusions}

The model for Wolf-Rayet stars considered by many, see Section 1, to be appropriate is supported neither by reliable analysis of the spectra of Wolf-Rayet stars nor by the properties of eclipsing spectroscopic binaries containing Wolf-Rayet stars.

The model put forward by Underhill, see Section 1 , is responsive to the results of spectrum analysis and to the results from eclipsing spectroscopic binaries containing WolfRayet stars. It is a viable proposition to consider Wolf-Rayet stars to be massive premainsequence stars still surrounded by the remnant of a natal disk. Small magnetic fields appear to be present in the disk with the result that MHD effects create the high electron temperatures which Wolf-Rayet spectra imply.

The location of Wolf-Rayet stars in a galaxy indicates those regions where massive stars have formed in the presence of larger than normal interstellar magnetic fields. If these magnetic fields were not present, the newly formed stars would show spectral types 
in the range B1-08. Wolf-Rayet spectra imply high $T_{e}$ in the LER and moderate $T_{*}$. This state of affairs is a result of the deposit of non-radiative energy in the LER surrounding the star. It is not a normal result of stellar evolution.

\section{References}

Bhatia, A. K., and Underhill, A. B. 1986, Ap. J. Suppl., 60, 323. (BU86)

Bhatia, A. K., and Underhill, A. B. 1988, Ap. J. Suppl., 67, 187. (BU88)

Bhatia, A. K., and Underhill, A. B. 1989, Ap. J., submitted. (BU89)

Castor, J. I., and van Blerkom, D. 1970, Ap. J., 161, 485.

Drissen, L., Moffat, A. F. J., Bastien, P., Lamontagne, R., and Tapia, S. 1986, Ap. J., 306, 215.

Drissen, L., St. Louis, N., Moffat, A. F. J., and Bastien, P. 1987, Ap. J., 322, 888.

Langer, N. 1989, Astr. Ap., $210,93$.

Maeder, A., and Meynet, G. 1987, Astr. Ap., 182, 243.

Maeder, A., and Meynet, G. 1988, Astr. Ap. Suppl. Ser., 76, 411.

Moffat, A. F. J., Drissen, L., Lamontagne, R., and Robert, C. 1988, Ap. J., 334, 1038.

Pudritz, R. E., and Norman, C. A. 1986, Ap. J., 301, 571.

Schulte-Ladbeck, R. E., and van der Hucht, K. A. 1989, Ap. J., 337, 872.

Stickland, D. J., Bromage, G. E., Budding, E., Burton, W. M., Howarth, I. D., Jameson, R., Sherrington, M. R., and Willis, A. J. 1984, Astr. Ap., 134, 45.

St. Louis, N., Drissen, L., Moffat, A. F. J., and Bastien, P. 1987, Ap. J., $322,870$.

St. Louis, N., Moffat, A. F. J., Drissen, L., Bastien, P., and Robert, C. 1988, Ap. J., 330, 286.

Underhill, A. B. 1983, Ap. J., 266, 718.

Underhill, A. B. 1984, Ap. J., 276, 583.

Underhill, A. B., Gilroy, K. K., and Hill, G. M. 1990, Ap. J., 351, 651.

Underhill, A. B., Gilroy, K. K., Hill, G. M., and Dinshaw, N. 1990, Ap. J., 351, 666. 


\section{DISCUSSION}

Niemela: There are also disks around evolved stars, this could be the case for WR stars. Underhill: I postulate that WR stars are young stars just approaching the ZAMS. The disks around cataclysmic variables have a different origin. Those around WR stars are the remnant of the natal cloud; those around evolved stars are from a companion.

Montmerle: Could you give more details on the analogy you make between WR stars and $\mathrm{T}$ Tauri stars? There is no evidence for disks around T Tauri stars from their emission-line spectra; thus evidence comes - indirectly - mostly from their IR excess. Their emission lines are thought to come from a boundary layer between the star and the accretion disk, not from the disk itself.

Underhill: The T Tauri lines have similar shapes as WR lines, mostly a broad rather flat-topped emission feature. Such lines can be formed in rotating turbulent disks, see the numerical experiment of Underhill and Nemec (1989). Also the energy in T Tauri emission lines is $0.1-0.3 L$ as in WR stars, and the electron temperature in the line-emitting region is higher than in the photosphere in both types of stars. Some investigators suggest the line emission of $\mathrm{T}$ Tauri stars comes from a wide ring-like disk. This is what I think is possible for WR stars. The disk is a remnant of the birth cloud.

Maeder: If WR stars would be pre-MS stars, their number ratio to $\mathrm{O}$ stars should be about 0.01 rather than 0.1 . Of course, I know your argument that WR stars originate in a relatively low mass range, but then one should not only find them in very young clusters. Underhill: I believe the number of WR stars is no more than 0.01 of all the $O$ stars in the Galaxy. My point is that WR stars are found only where there is massive star formation $\left(M>10 M_{\odot}\right)$, but also weak interstellar magnetic fields must be present to create the necessary weak magnetic field which in turn permits mechanical energy to be transformed to heat energy. This results in the high electron temperature needed to generate a WR-type spectrum.

Dopita: How do you explain the relative paucity of WR stars in low-metallicity systems which are seen to be undergoing a higher specific star formation than, for example, our galaxy?

Underhill: According to my scenario WR stars form only in those molecular clouds where larger than normal interstellar magnetic fields are present.

Dopita: The magnetic field in the LMC appears to be exactly the same as in the solar region.

Underhill: The magnetic field in the LMC surely is not the same everywhere.

Cassinelli: The difficulty that $I$ have with the pre-main sequence model is that WR stars do not have the properties of known pre-main sequence massive stars. The ultracompact $H I I$ $(U C H I I)$ regions that have been studied by Churchwell and Wood are clearly young $\mathrm{O}$ stars deep in their natal molecular cloud. These objects are heavily extincted, unobservable at optical wavelengths and have infrared excesses from surrounding dust cocoons. In contrast WR stars often have small $E(b-v)(\lesssim 0.1)$, and have a moderate IR excess that can be explained by free-free emission from the wind.

Underhill: A Wolf-Rayet spectrum of emission lines and $\mathrm{f}-\mathrm{f}$ continuum from a disk-like lineemitting region can be seen only after the dust and other material has dissipated. According to my scenario, WR stars are practically on the ZAMS; their lifetimes as emission-line stars may be as little as $10^{4}-10^{5}$ years. Many are heavily reddened, possibly in part by the remnant of a cocoon. 